\title{
Phase separation of an Ionic Liquid mixture assisted by a temperature gradient.
}

\author{
Marc Pascual, Arthur Poquet, and Alexandre Vilquin \\ Gulliver CNRS UMR 7083, PSL Research University, \\ ESPCI Paris, 10 rue Vauquelin, 75005 Paris, France \\ Marie-Caroline Jullien* \\ Gulliver CNRS UMR 7083, PSL Research University, \\ ESPCI Paris, 10 rue Vauquelin, 75005 Paris, France and \\ Université Rennes 1, CNRS, IPR (Institut de Physique de Rennes) - UMR 6251, F-35000 Rennes, France
}

(Dated: February 18, 2021)

Ionic liquids have remarkable properties and are commonly harnessed for green chemistry, lubrication and energy applications. In this paper, we study a thermo-responsive Ionic Liquid (IL) solution which has the property of phase separating above a critical temperature, an interesting feature for the recovery of the IL-rich phase. For this purpose, we generate a temperature gradient in a microfluidic cavity where the confinement strengthens wetting effects and enhances the demixing. We show that the phase separation is performed by the joint effects of sedimentation and thermocapillary actuation giving rise to a $3 \mathrm{D}$ flow structure, which is quantitatively captured by our model. Altogether those mechanisms lead to the accumulation of the wetting phase near the heating source. We believe this work will find applications in the recycling of ionic liquids.

\footnotetext{
* marie-caroline.jullien@univ-rennes1.fr
} 


\section{INTRODUCTION}

Last decade, temperature-responsive binary systems made of ionic liquids (IL) and water have shown promising applications such as in metal and rare-earth extraction [1, 2], protein stabilisation [3, 4], catalytic synthesis [5, 6], forward osmosis water desalination [7-9] and low grade heat valorization [10, 11]. Those techniques are built on the reversible phase separation of the system with temperature loops. As ionic liquids are costly and generally available in limited volumes, isolating separately the two phases is crucial. This issue has rekindled the interest of phase-separation widely studied since the $70 \mathrm{~s}$, both experimentally and theoretically [12]. Besides the industrial centrifugation techniques or the sedimentation mechanisms [13, 14], microfluidics appears as an excellent alternative since it enhances capillary mechanisms, especially when wetting effects, which are marked in binary mixtures [15-17], are known to boost the phase-separation kinetics of such systems [18-24]. As an example, the use of a confinement gradient is an interesting method where separation relies on a well understood capillary effect [25]: the most wetting phase is dragged toward the wedge, i.e. toward the thinner part of the cavity. Such experiments have been performed (see Supplemental Movie S1) and the separated phases are recovered in two well-defined regions. This wetting-based strategy is expected to occur up to confinement below the interfacial capillary length $\left(l_{c}=\sqrt{\gamma / \Delta \rho g} \approx 300 \mu \mathrm{m}\right.$ at $\left.35^{\circ} \mathrm{C}\right)$. As the physics at play are understood, the results are not discussed here.

In this paper, we present a strategy that takes advantage of Marangoni effects at small scales. A temperature-induced interfacial tension gradient is known to cause an interfacial stress generating thin film flow [26-29] and droplets or colloids motion [30-35], thereby affecting the phase separation kinetics [36]. Note that concerning the phase transition of a pure fluid, the mechanisms of droplets or bubbles migration in a temperature gradient are not induced by Marangoni stresses [37, 38]. Previous studies on the phase separation of binary mixtures in a temperature gradient focused on bulk migration: drop migration assisted by thermocapillarity [39, 40], or drop sedimentation under gravity $[13,14]$. We propose an alternative approach that combines sedimentation and thin film interfacial flow. The coupling of surface and volume convection, generating a complex three-dimensional flow structure, is crucial in the separation dynamics. Together, they lead to remarkable phase separated patterns. The mechanisms are first detailed using an IL-water mixture, and their universality in term of wetting properties is validated with another well-known binary system: the lutidine-water mixture.

\section{MATERIALS \& METHODS}

The microfluidic system is sketched in Fig. 1(a). The polydimethylsiloxane cavity is replicated from a micromilled brass mold and bonded on a glass coverslip with oxygen plasma treatment. The cavity is a cylinder of $1 \mathrm{~cm}$ in diameter whose height ranges from 20 to $200 \mu \mathrm{m}$. Its bottom surface is layed onto a glass wafer with micropatterned resistance $\left(50 \times 500 \mu \mathrm{m}^{2}\right)$, electrically isolated by spincoating a $30 \mu \mathrm{m}$ PDMS layer [41]. The potential applied to the heating resistor can be tuned up to 10 Volts. Phase separation dynamics is observed using bright-field transmitted light video microscopy.
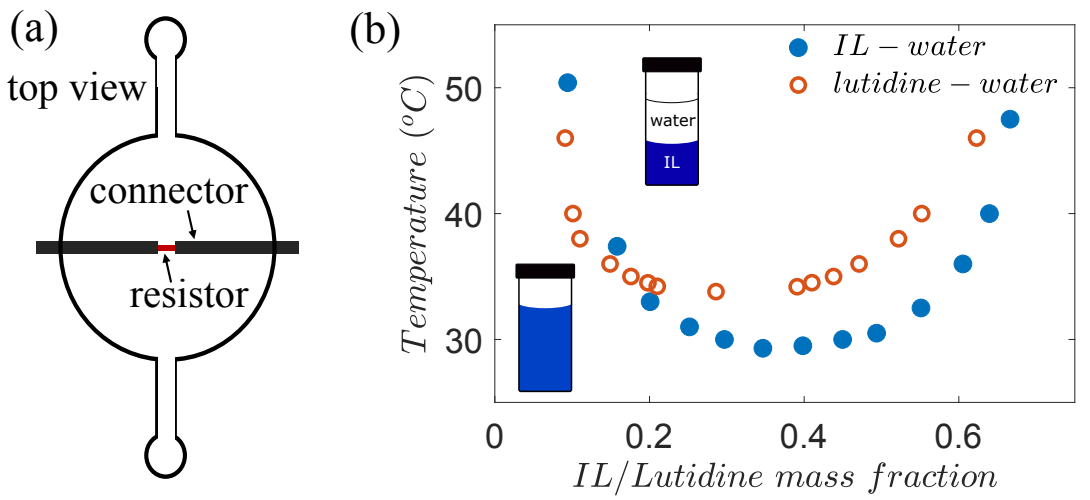

FIG. 1. (a) Top view of the microfluidic cavity $1 \mathrm{~cm}$ in diameter. The gold connectors are in dark grey, and the chromium resistor is in red $\left(50 \times 500 \mu^{2}\right)$. (b) Phase diagram of the IL-water (blue dots) and lutidine-water systems (orange circles). The two sketches show the homogeneous and separated states of the mixture. The IL-rich is denser and colored with a blue dye. 
The main binary system under investigation is a mixture of MilliQ water with the ionic liquid tetrabutylphosphonium trifluoroacetate $\left(P_{4444} C_{3} F_{3} O_{2}\right)$ [42, 43] already used in different applications [7, 8, 10], at an IL mass fraction of $25 \%$ (separation temperature $T_{s e p}=31^{\circ} \mathrm{C}$ ). This system is known as a Lower Critical Solution Temperature (LCST) binary solution, i.e. it phase separates above a critical temperature (see Fig. 1(b)). The interfacial tension of LCST mixtures increases with temperature and as a consequence the thermocapillary stress is directed toward the warmer region. Fig. 2(a) displays the measurements of the interfacial tension between the two separated phases versus the temperature of the mixture. They are performed in rising drop configuration (PSA30-Kruss), a circulating water-bath (Julabo HL-4) sets the temperature of the sample. The data of the IL-water mixture are compared to lutidine-water data from the literature [44].

The temperature mapping is performed independently using a temperature sensitive fluorescent solution (rhodamineB, Sigma-Aldrich, $50 \mathrm{mg} . \mathrm{L}^{-1}$ ) [45]. We did not achieve to measure the temperature in the cavity during a phaseseparation experiment: the rhodamine fluorescence is affected by the elevated Ionic Liquid concentration. The calculated Péclet number, $P e=\left(H v_{r}\right) / \alpha_{T} \approx 0.1$ with $H=100 \mu \mathrm{m}, \alpha_{T}=1.5 .10^{-7} \mathrm{~m} \cdot \mathrm{s}^{-2}$ and $v_{r}=150 \mu \mathrm{m} \cdot \mathrm{s}^{-1}$ (see Fig. 6 for typical velocities), shows that the impact of convection on the temperature profile can be neglected. Moreover IL-water mixtures at $25 w t \%$ and rhodamine solutions have similar heat capacity and thermal diffusivity. Thus, we fill the same cavity (among others the same resistance) with a rhodamine solution and apply the same voltage. Once the voltage is applied to the resistor, the temperature suddenly increases at the resistor location. At early time, i.e. in the first second (see discussion on $r_{\text {out }}$ in section III.A), the temperature diffuses above the resistor displaying a $3 \mathrm{D}$ profile in the microfluidic cavity. After this transient stage, the temperature profile can be considered as $2 \mathrm{D}$, i.e. diffuses radially along the cavity, and writes $T(r, t)$. Fig. 2(b) displays the radial temperature profiles at different times and the inset shows the same curves rescaled by $\sqrt{\alpha_{T} t}$, where $\alpha_{T}=1.5 \times 10^{-7} \mathrm{~m}^{2} . \mathrm{s}^{-1}$ is the thermal diffusivity of water. All profiles collapse on a single curve proving the 2D diffusion of temperature along the cavity with time.
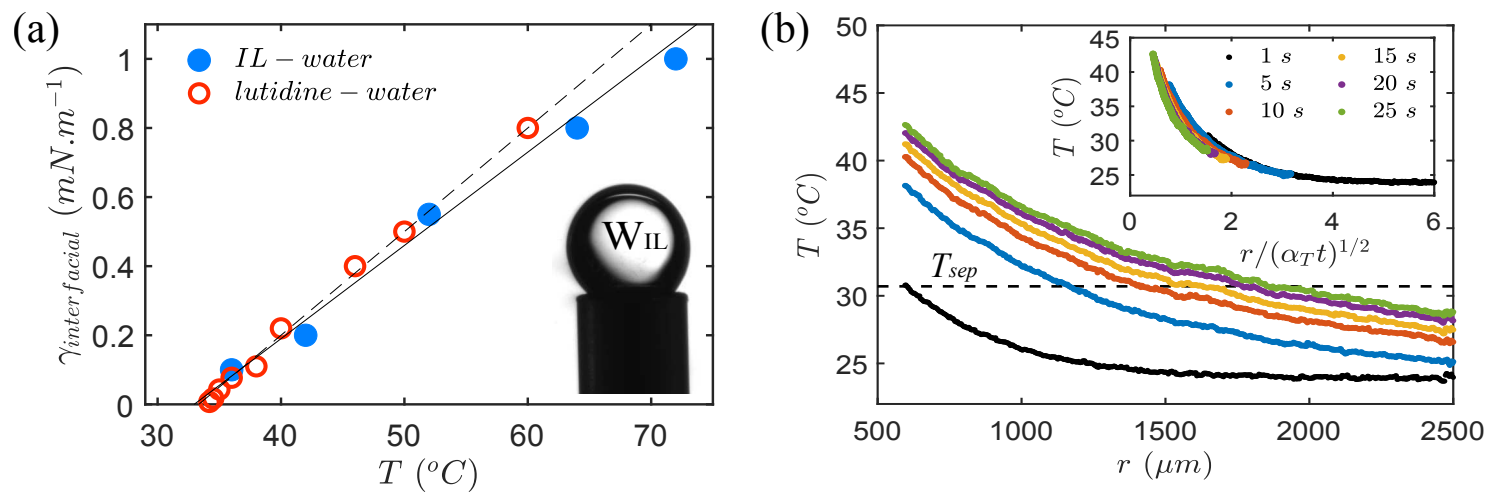

FIG. 2. (a) Interfacial tension between the IL-rich (lutidine-rich) and the water-rich phases as a function of the temperature. The solid line gives $\partial_{T} \gamma=2.5 \times 10^{-5} \mathrm{~N} \cdot \mathrm{m}^{-1} . \mathrm{K}^{-1}$ for the IL-water system, the dashed line gives $\partial_{T} \gamma=3.0 \times 10^{-5} \mathrm{~N} . \mathrm{m}^{-1} . \mathrm{K}^{-1}$ for the lutidine-water system (adapted from ref [44]). Inset: picture of the water-rich rising drop embed in a thermostated IL-rich bath. (b) Temperature profile in the cavity for six different times. The radial coordinate is the distance to the resistor center. The black dashed horizontal line indicates $T_{s e p}$. Inset: temperature profile with the radial coordinate rescaled by a thermal diffusion lengthscale. The applied voltage is 7 Volt $(80 \mathrm{~mW})$.

As for the dynamics of binary mixtures under homogeneous temperature, the initial composition of the solution imposes the phase separation pattern and kinetics $[46,47]$. In the following, we focus on the case where the wetting phase (the IL-rich phase here) is the dispersed phase, i.e. the IL mass fraction is smaller than $35 \%$ (consolute point of the mixture). The other system is a mixture of MilliQ water with 2,6-lutidine (Sigma Aldrich) at $40 \mathrm{wt} \%$ in lutidine $\left(T_{\text {sep }}=34^{\circ} \mathrm{C}\right)$. In this case the wetting phase (the water-rich phase as the lutidine-rich phase is non-wetting with the plasma treated chips) is also the dispersed phase. The separation curves of both systems are displayed on Fig. 1(b).

\section{RESULTS}

\section{A. Description of the separation pattern}

The temperature diffusion triggers the phase separation which propagates from the center towards the edges. The separation temperature $\left(T_{s e p}\right)$ isotherm is directly visible at the front of the dark cloud stemming from the light scattering of the dense two phase system, whose droplets sizes are much smaller than the cavity thickness. 
Qualitatively, the droplets size distribution in this cloud is not monodisperse as the droplets undergo ageing from the outside to the inside. The temperature steady state is reached after $6 \pm 3$ minutes depending on room temperature.

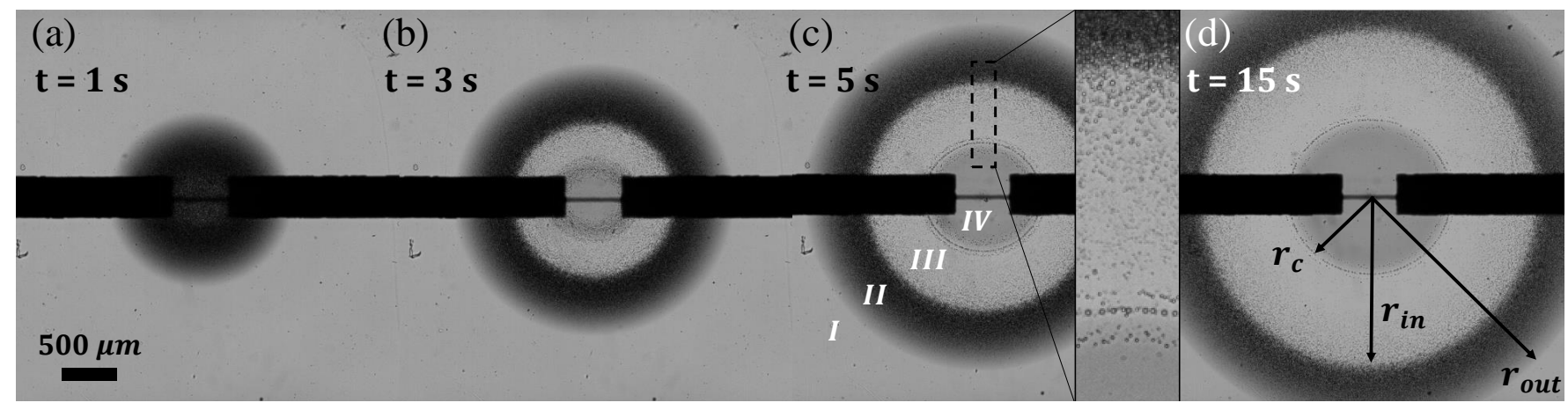

FIG. 3. (a)-(d) Phase separation timelapse of the IL-water solution at 7 Volts $(80 \mathrm{~mW})$. The mass fraction in IL is $25 \%$ and the cavity height is $H=60 \mu \mathrm{m}$. (c) Definition of the four regions within which the flows are rationalized: I) IL-water non-separated mixture, II) Separation of the two phases, the droplets are mostly pushed outwards, III) some IL-rich droplets are dragged inward across the water-rich phase, IV) IL-rich sessile drop. Inset: Zoom on the droplets moving from the cloud to the center drop. (d) Definition of $r_{c}, r_{i n}$ and $r_{\text {out }}$, corresponding to the central drop radius, along with the cloud inner and outer radii.

For the whole span of cavity heights we observe the following behavior: the opaque cloud of droplets appearing above the resistor expands isotropically in $2 \mathrm{D}$ with the $T_{\text {sep }}$ isotherm diffusion (Fig. $3(\mathrm{a})$ ): this front is tracked as $r_{\text {out }}$ following a square root dependence with time ( $c f$. Fig. 4). The cloud opens up in its center (Fig. 3(b)) to reveal a darker IL-rich drop of radius $r_{c}$, and in-between this drop and the cloud, a few droplets come back toward the central drop from $r_{i n}$ (Fig. 3(b)-(d)). To clarify our explanation, the cavity is divided into four regions: $I$ the region located outside the isotherm $T_{\text {sep }}$ in which the binary mixture is not separated yet $\left(r>r_{\text {out }}\right), I I$ the region within which the mixture is phase separated and the droplets move outwards following the temperature diffusion front $\left(r_{i n}<r<r_{\text {out }}\right)$, $I I I$ the region enriched in water in which small droplets are driven inwards $\left(r_{c}<r<r_{i n}\right)$, and $I V$ the region enriched in IL $\left(r<r_{c}\right)$. Surprisingly, the droplets of regions $I I$ and $I I I$ are driven in opposite sides (see Supplemental Movie $\mathrm{S} 2 \& \mathrm{~S} 3)$.

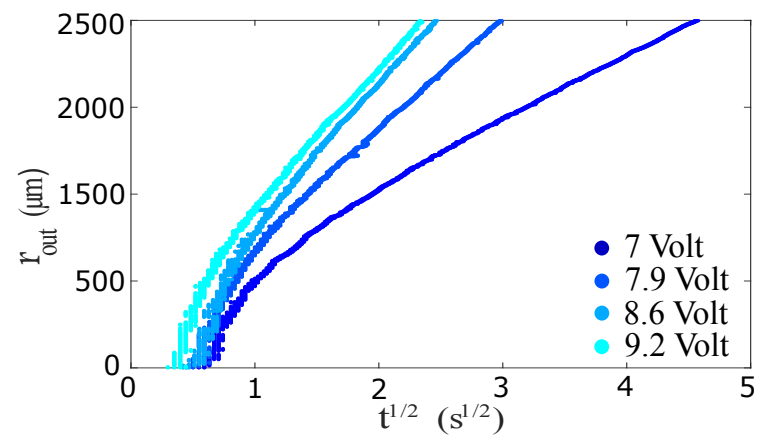

FIG. 4. Position of the cloud outer radius $r_{\text {out }}$ versus the square root of time. Voltages applied are 7, 7.9, 8.6 and 9.2 Volts $(R=620 \Omega)$. Cavity Height is $H=60 \mu \mathrm{m}$. IL mass fraction is $25 \%$.

Fig. 4 represents the temporal evolution of the cloud outer radius $r_{\text {out }}$ which coincides with the propagation of the separation temperature isotherm $T_{\text {sep }}$. As this evolution is due to quasi $2 \mathrm{D}$ temperature diffusion in the cavity, we recover a square root dependence with time (except for shorter times before $1 \mathrm{~s}$, where we observe the signature of the 3D thermal diffusion). Nevertheless the direct comparison of $r_{\text {out }}(t)$ with the temperature profile evolution is tricky since $\mathrm{a} \pm 1^{\circ} \mathrm{C}$ variation gives an uncertainty of $\pm 500 \mu \mathrm{m}$ on the radial position. The higher the voltage (and the heat injected in the system), the faster the isotherm propagates. The upper limit $r_{\text {out }}=2.5 \mathrm{~mm}$ corresponds to the accessible visual window. At longer times, the temperature profile reaches a stationary state and $r_{\text {out }}$ tends to a constant (this regime is not displayed). 


\section{B. Flow profile in the cavity}

In order to understand why the droplets of regions $I I$ and $I I I$ are driven in opposite directions, it is necessary to tackle the 3D structure of the flow. This is possible using confocal microscopy (Leica DMi8 with scanning laser head TCS SP8) [48] allowing direct visualization in a cross-section of the cavity (see Supplemental Movie S4). Fluorescein (Sigma-Aldrich) is added at 0.1 g.L $\mathrm{L}^{-1}$ to the $25 \mathrm{wt} \%$ IL solution. The microscope records the fluorescence of excited fluorescein within the IL-rich phase, while the water-rich phase is dark. Fig. 5(a)-(d) displays fluorescent images of the same cross-section in the cavity at different times. The front of the phase separation propagates (Fig. 5(a)), IL-rich droplets nucleate, grow in size and sediment (as the denser phase) (Fig. 5(b)). Their complete spreading on the bottom gives birth to a micrometer-thick wetting layer which thickness $h$ grows rapidly to yield a value of $6 \pm 2 \mu \mathrm{m}$ throughout region $I I I$ (Fig. 5(b)-(c)). Some IL-rich droplets are conveyed above the film toward the center (Fig. 5(c)-(d)) and meet with the IL-rich central drop expanding (Fig. 5(d)).

Fig. 5(e) summarizes this 3D flow sketching the role of the film in region $I I I$ : as the surface tension increases with temperature, a thermocapillary stress drives the interface of the bottom IL film toward the center of the cavity. Thanks to momentum diffusion, this interfacial transport drags the water-rich phase as well as the IL-rich droplets located just above. The intake of IL-rich phase from the film accumulates at the center where a single IL-rich drop grows in size (region $I V)$. The central drop profile can be sessile (Fig. 3(b)-(d)) or wetting-disk type (Fig. 5(d)). We believe that the sessile profile is out of equilibrium and is sustained by thermal interfacial stresses since the IL is completely wetting. Moreover a sessile drop could not exist in the cavity since the capillary length $l_{c}$ exceeds the confinement. When the apex of the sessile drop makes contact with the upper wall, the drop rearranges in a wetting disk configuration bridging cavity lower and upper walls. By mass conservation, the water-rich phase converging to the center is sent back outwards and pushes aside the cloud of droplets.

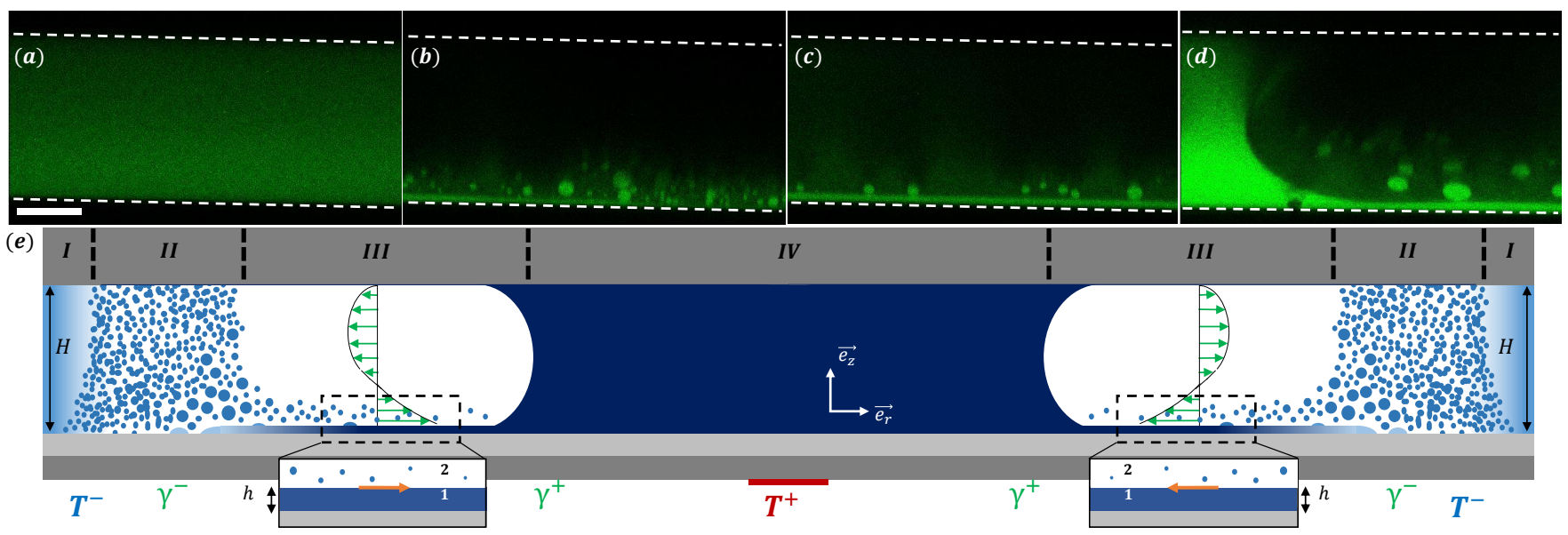

FIG. 5. (a)-(d) Confocal imaging of the phase separation along the height of the cavity at different times for 8 Volts (100 mW). The central drop seen on (d) is bridging top and bottom cavity walls. Scale bar is $40 \mu \mathrm{m}$. Cavity height is $H=120 \mu \mathrm{m}$. (e) Cross-section sketch of the phase separation dynamics. The tread-milling wetting film induces a radial Couette-Poiseuille flow. The regions $I, I I, I I I$ and $I V$ delimit respectively the non-separated solution, the droplet cloud, the Couette-Poiseuille flow induced by the film, and the central IL-rich drop. The red rectangle respresents the heating resistor. Inset: within region III, domain 1 indicates the IL-rich wetting film and domain 2 the water-rich phase above.

We now show that it is possible to rationalize this scenario and find quantitative agreement between our measures and the model. Experimentally the velocity field is radial and, as the vertical length scales $h$ and $H$ are much smaller than $r_{c}$, only the variation along $z$ is considered. The dependence on $r$ is contained in the interfacial stress $\partial_{r} \gamma$. Then the flow is assumed to follow the 2D Stokes equation in the lubrication approximation in a plan $(r, z)$ as $h \ll H$, see Appendix C. Region $I I I$ is split in two domains to solve the equation: domain 1 refers to the IL-rich wetting film at the bottom, domain 2 refers to the upper phase enriched in water (Fig. 5(e)insets). The Stokes equation in domain $1, \eta_{I L} \partial_{z}^{2} v_{1}=0$, is solved using $v_{1}(0)=0$ and the interfacial stress continuity at $z=h$ : $\eta_{I L}\left(\partial_{z} v_{1}\right)_{z=h}-\eta_{w}\left(\partial_{z} v_{2}\right)_{z=h}=\partial_{r} \gamma$. In this domain, we neglect the radial pressure term in the above Stokes equation: as $h \ll H$ the velocity profile is well approximated by a linear Couette flow. Since the viscosity of the IL-rich phase is 5 times the viscosity of the water-rich phase: $\eta_{I L} \approx 5 \eta_{w}$, and the film thickness is 10 times smaller than the cavity 
thickness, we have $\eta_{I L}\left(\partial_{z} v_{1}\right)_{z=h} \gg \eta_{w}\left(\partial_{z} v_{2}\right)_{z=h}$. As such, the flow field in domain 1 yields:

$$
v_{1}\left(\partial_{r} \gamma, z\right)=\frac{\partial_{r} \gamma}{\eta_{I L}} z
$$

It is not possible to measure this velocity profile directly, nevertheless the droplets located just above the film are conveyed at a velocity with same order of magnitude thanks to viscous diffusion of momentum. These droplets can serve as tracers, noting that they move slower than the interface, until they eventually merge with the film. The temperature gradient range is $\partial_{r} T \in[0.52] \times 10^{4} \mathrm{~K} . \mathrm{m}^{-1}$, which gives the expected interfacial velocities $v_{1}(h) \in[150600] \mu \mathrm{m} . \mathrm{s}^{-1}$, with $\partial_{T} \gamma=2.5 \cdot 10^{-5} \mathrm{~N} . \mathrm{m}^{-1} \cdot \mathrm{K}^{-1}, \eta_{I L}=5.10^{-3}$ Pa.s and $h=6 \mu \mathrm{m}$. We plot on Fig. 6 the droplets velocities measured by PIV for 5 successive times [49]. The maximum speed reaches $300 \mu \mathrm{m} \cdot \mathrm{s}^{-1}$ at shorter times, showing good agreement with the order of magnitude of the calculated interfacial velocity. Flows stemming from buoyancy contribution are of typical velocity $0.008 H^{3} g \partial_{r} \rho / \eta_{w} \approx 5 \mu \mathrm{m} \cdot \mathrm{s}^{-1}$ and are thus found negligible [50, 51].

The linear distribution of the droplets velocities at each time is difficult to rationnalize since: 1) the droplets sediment throughout time and shall not keep the same distance from the film dragging them, 2) they are also submitted to thermocapillary action opposite to their direction of propagation and 3) they shrink in size while going up the temperature gradient because of composition equilibration (water is expelled from the IL-rich droplet). The sedimentation brings droplets closer to the film and should accelerate them even more. The contributions of thermocapillary action and droplet's size diminution should decrease the droplets speed. These contributions may balance each other since the thermocapillary force is proportionnal to $R \partial_{r} T$ (with $R$ the droplet radius): $\partial_{r} T$ increases as the droplet comes closer to the center but simultaneously $R$ decreases. We do not model these effects analytically in this work. Inspired by the work of Roché et. al. on interfacial Marangoni flows [52], the inset on Fig. 6 shows the rescaled velocity profiles $v_{r} / v_{\max }$ as a function of the reduced radial coordinate $\left(r_{i n}-r\right) /\left(r_{i n}-r_{c}\right)$. The collapse of the five profiles testifies that the droplets migration obeys the same mechanism and the above cited effects keep the same balance throughout time.

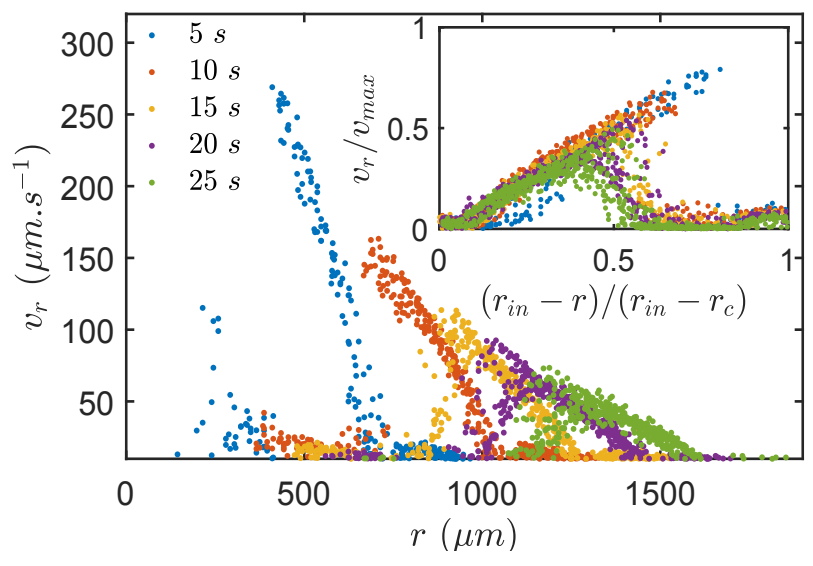

FIG. 6. Velocity $v_{r}$ of the droplets coming back to the center versus the radial coordinate, at five successive times: 5, 10, 15, 20 and $25 \mathrm{~s}$. The mass fraction in IL is $25 \%$, the cavity height is $H=60 \mu \mathrm{m}$ and the voltage is 7 Volts. Inset: rescaled velocity versus the reduced spatial coordinate. All profiles collapse on a line with a slope of 1 .

In domain 2 of region $I I I$, the water-rich phase swept to the center by the shear flow has no option but to turn back when it reaches the central drop, due to axial symmetry and mass flux conservation. This counter Poiseuille flow adds up with the thermocapillary effect to push the cloud of region II outward (Fig. 5(e)). Considering the same assumptions as in domain 1, the flow in domain 2 is described by the Stokes equation: $\eta_{w} \partial_{z}^{2} v_{2}=\partial_{r} p$, which is solved using $v_{2}(H)=0, v_{1}(h)=v_{2}(h)$ and the flux conservation $\int_{0}^{h} v_{1}(z) d z+\int_{h}^{H} v_{2}(z) d z=0$, leading to:

$$
v_{2}\left(\partial_{r} \gamma, z\right)=\frac{\partial_{r} \gamma}{\eta_{I L}} \frac{h(H-z)}{(H-h)}\left(1-\frac{3 H(z-h)}{(H-h)^{2}}\right)
$$

Then the average velocity of the Poiseuille flow $v_{2}^{a v}$ can be safely calculated. 


\section{Pattern dynamics: $r_{i n}(t)$ and $r_{c}(t)$}

Consequently, the cloud inner radius $r_{i n}$ is expected to move at a velocity which results from the addition of this Poiseuille flow to the thermocapillary migration, given by $v_{T c a p}=\frac{R \partial_{T} \gamma \partial_{r} T}{3 \eta_{w}\left(1+3 \eta_{I L} / 2 \eta_{w}\right)}[53]$ :

$$
\partial_{t} r_{i n}(t)=v_{2}^{a v}(t)+v_{T c a p}(t)
$$

Thereby the temporal dependence of $\partial_{t} r_{i n}$ is mainly carried by $\partial_{r} \gamma\left(r=r_{i n}(t)\right)$. The confocal imaging allows us to estimate $h \simeq 3 \mu \mathrm{m}$ at $r=r_{i n}$ and $R \simeq 10 \mu \mathrm{m}$ for the cloud droplets radii, which we set as constants over time (see Appendix C). Given these values, Fig. 7(a) displays the calculated Poiseuille contribution as a dashed line, and the sum of the Poiseuille and the thermocapillary migration as a solid line. Remarkably, this model reproduces the experimental data plotted as dark crosses showing the relevance of our model. In order to evaluate the sensitivity of the velocities on $h$ and $R$, a variation of $30 \%$ is represented as lighter blue zones in the plots.

(a)

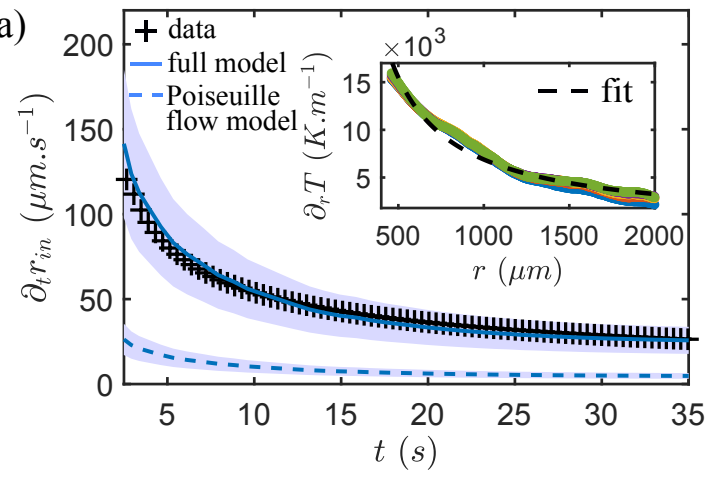

(b)

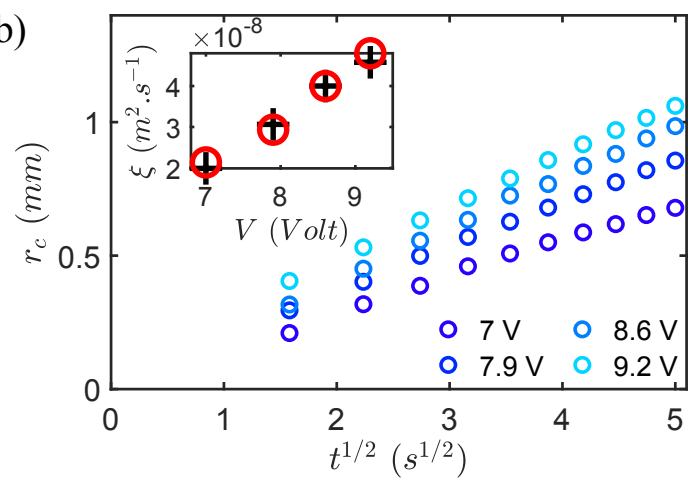

FIG. 7. (a) Measured velocity of the cloud inner radius $\partial_{t} r_{i n}$ versus time, at 7 Volt (black crosses). The blue dashed line represents the average velocity of the Poiseuille contribution eq.(2). The blue solid line is the sum of the Poiseuille and thermocapillary contributions eq.(3). The blue shaded areas give a confidence interval related to a $30 \%$ variation in $h$ and in $R$. Inset: Temperature gradient evolution in the cavity at 5, 10, 15, 20 and 25 seconds after switch-on, derived from the temperature profiles. The black dashed line is a $1 / r$ fit. (b) Growth dynamics of the central drop radius $r_{c}=(\xi t)^{1 / 2}$ at four voltages. Inset: Comparison between calculated $\xi$ (red circles) and experimental one (black crosses), see eq.(4).

Finally we explain the dynamics of the central drop radius $r_{c}$ plotted on Fig. 7(b). This drop is assumed to be a flat cylinder of volume $V_{c}=\pi r_{c}^{2} H$ which is filled by the flux coming from the IL-rich wetting film. The mass conservation leads to $\partial_{t} V_{c}=2 \pi r_{c} \int_{0}^{h} \frac{\partial_{r} \gamma}{\eta_{I L}} z d z$. Assuming that the temperature gradient is inversely proportional to $r, \partial_{r} T=\Delta T / r$ (cf. Fig. 7(a)inset, this feature is discussed in Appendix A), we get:

$$
r_{c}(t)=\left(\frac{\partial_{T} \gamma \Delta T h^{2}}{H \eta_{I L}}\right)^{1 / 2} t^{1 / 2}=(\xi t)^{1 / 2}
$$

The inset of Fig. 7(b) compares the experimental and calculated values of the prefactor $\xi$ for which we find good agreement without fitting parameter. Given the uncertainties yielded by $\Delta T$ and $h$, the confidence interval for $\xi$ is within $20 \%$ of its value. As a whole, our model supports the scenario described previously. The data shown here correspond to a Voltage of 7 Volts. The measurements obtained for other voltages are gathered in Appendix B.

\section{Late stage of the dynamics}

With $r_{i n}$ being propelled by both the Poiseuille flow and the thermocapillary migration, $\partial_{t} r_{i n}$ is driven by a different mechanism than $\partial_{t} r_{\text {out }}$. Hence the inner radius of the toroidal cloud can propagate faster than the $T_{\text {sep }}$ isotherm, and $r_{\text {in }}$ may overtake $r_{\text {out }}$. It occurs after 10 to 100 seconds depending on the room temperature: the colder it is, the sooner $r_{\text {out }}$ starts to reach steady-state and the sooner $r_{\text {in }}$ catches up. When it happens the cloud vanishes gradually, the number of phase-separated IL-rich droplets diminishes drastically and the wetting layer suffers from a supply shortage in IL. The continuous drainage to the center thins the wetting film and triggers a periodic destabilization: 
the film configuration switches into several fingers (see Fig. 8). Along a finger the interfacial shear flow keeps dragging material from region $I$ toward the central region. By doing so, the material starts to phase separate as the temperature rises, producing a visible darker plume composed of IL-rich droplets superimposed to the wetting film finger (see Supplemental Movie S5).

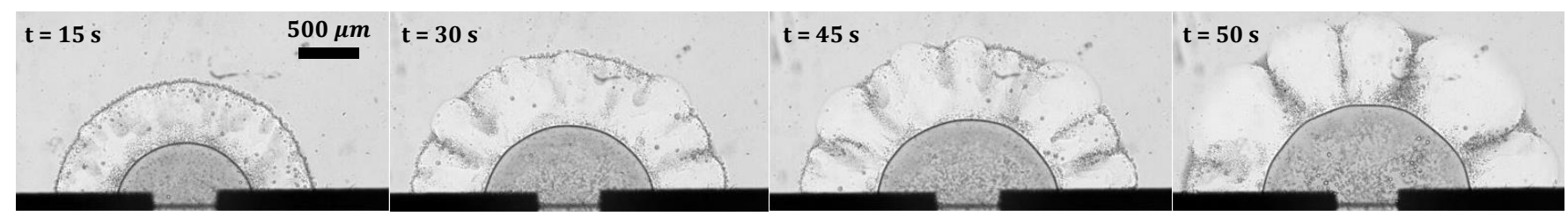

FIG. 8. Destabilisation of the wetting film into periodic digitations as $r_{\text {in }}$ catches up $r_{\text {out }}$. The central IL-rich drop is wetting both cavity floor and ceiling. The applied voltage is 8 Volt $(100 \mathrm{~mW})$ and the cavity height is $H=80 \mu \mathrm{m}$.

This digitation pattern is maintained as long as the isotherm of phase separation $T_{\text {sep }}\left(i . e . r_{\text {out }}\right)$ keeps on propagating, it can last a few minutes. When $T_{\text {sep }}$ becomes static the fingers gradually disappear and we end up with the final state of the dynamics: the IL-rich wetting phase has accumulated at the center above the heating resistor while the water-rich non-wetting phase is distributed all around. The radius $r_{\text {out }}$ of the whole pattern is given by the position of $T_{\text {sep }}$. The complete study of this instability is out of the scope of the paper. When $r_{\text {out }}$ exceeds the radius of the cavity, all the liquid in the cavity is phase separated. Increasing even more the temperature induces further concentration of both phases in their respective majority constituents (according to the phase diagram in Fig. 1(b)).

\section{E. Lutidine-water system}

In order to tackle the reproducibility of the demixing pattern regarding the role of the wetting phase, we performed the same experiments using another LCST binary mixture widely employed in the literature: the lutidine-water system. To do so, a binary solution with a weight fraction of $40 \%$ in Lutidine $\left(T_{\text {sep }}=34^{\circ} \mathrm{C}\right)$ is injected in the oxygen plasma treated cavity, such that water becomes the dispersed phase expected to develop a wetting film on the hydrophilic walls [54].

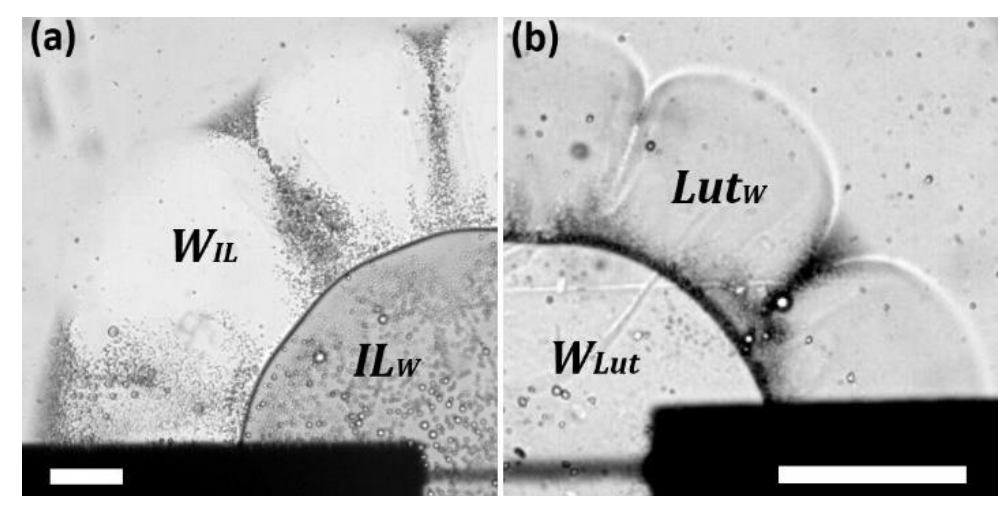

FIG. 9. Phase separation patterns of: (a) IL-water, (b) lutidine-water mixtures in a hydrophilic cavity. Mass fraction in IL and lutidine are respectively $25 \%$ and $40 \%$ : in both cases the wetting phase is the dispersed component. Images are taken respectively 55 and 10 seconds after switch-on. The applied voltage is 8 Volt $(100 \mathrm{~mW})$ and the cavity height is $H=80 \mu \mathrm{m}$. In both cases the cloud has vanished and the wetting film already destabilised to form digitations. On the left the central drop is the IL-rich phase $\left(I L_{W}\right)$, while it is the water-rich phase $\left(W_{L u t}\right)$ on the right. Scale bars are $300 \mu \mathrm{m}$.

Fig. 9 compares the demixing patterns of our two systems: IL-water on the left and lutidine-water on the right. In both cases the picture is taken when the wetting film has destabilized to form fingers. Qualitatively, the higher separation temperature of our lutidine-water mixture implies a slower propagation of $r_{\text {out }}$ compared to the IL-water experiments (the higher the separation temperature, the slower its isotherm propagates) and the destabilization of the wetting film happens at shorter times (the propagation pace of $r_{i n}$ is tuned by the temperature gradient: a greater difference between room and resistor temperature implies a steeper gradient). Nevertheless the two patterns on Fig. 9 look very similar. As expected the water-rich phase behaves like the IL-rich phase discussed previously: 
the digitations cross the surrounding lutidine-rich phase and water is accumulated above the heating resistor. These results comfort the universality, in term of the wetting properties, of the demixing scenario.

\section{CONCLUSION}

To conclude, we investigated the influence of a temperature gradient on the phase separation of LCST binary mixtures under confinement. Same as the experiments carried with homogeneous heating, the volume fraction and wetting behavior of both constituents drive the dynamics. The emergence of a micrometric wetting film submitted to thermal interfacial tension gradients is responsible for strong flows directed toward the warmer region. We rationalized the 3D complex flow generated by the separation and found good quantitative agreement with experimental measures. More importantly, the wetting phase carried in the film is entirely accumulated at the warmer side. Thus, tuning the wall surface energy allows to select which component shall be concentrated and eventually recovered. This behavior should be universal to all LCST binary mixtures. The present paper paves a way in binary mixture recycling. The impact of UCST behavior and spatial patterning in the cavity will be the object of future works [55-58].

\section{ACKNOWLEDGMENTS}

The authors are grateful to L. Bocquet for driving them towards this subject, to E. Martin for his help with the micromilling device, and wish to thank M. Kerdraon, A. Mansur, L. Keiser, J. T. Stockmann, J. D. McGraw, D. Beysens and L. Leibler for fruitful discussions. This work was supported by CNRS, ESPCI Paris, Agence Nationale de la Recherche (ANR) under the grant ANR-18-CE09-0029, IPGG (Equipex ANR-10-EQPX-34 and Labex ANR10-LABX-31) and PSL (Idex ANR-10-IDEX-0001-02).

\section{APPENDIX A: TEMPERATURE PROFILE IN THE CAVITY}

We legitimize here the use of $\partial_{r} \gamma=\Delta T / r$ in the paper. A simple model to estimate the dependence of $T$ is to consider the heat diffusion equation between two concentric cylinders of radii $r_{c}$ and $r_{i n}>r_{c}$, held at two temperatures $T_{c}$ and $T_{i n}$. Since $r_{c}$ and $r_{i n}$ both follow a square-root dependence with time, we argue that $T_{c}$ and $T_{i n}$ are rather constants and their value is determined by the y-axis values: $T_{c}=T\left(r_{c}(t) /\left(\alpha_{T} t\right)^{1 / 2}\right)$ and $T_{i n}=T\left(r_{i n}(t) /\left(\alpha_{T} t\right)^{1 / 2}\right)$ on Fig. 2(b)inset. Then the heat diffusion equation between moving boundaries $r_{c}(t)$ and $r_{i n}(t)$ is stationary and we have:

$$
\frac{1}{r} \frac{\partial}{\partial r}\left(r \frac{\partial T}{\partial r}\right)=0
$$

which gives after integration:

$$
T(r, t)=T_{c}-\frac{T_{c}-T_{i n}}{\ln \left(r_{i n}(t) / r_{c}(t)\right)} \ln \left(r / r_{c}(t)\right)
$$

So that the temperature gradient writes:

$$
\frac{\partial T}{\partial r}=-\frac{T_{c}-T_{i n}}{\ln \left(r_{i n} / r_{c}\right)} \frac{1}{r}=-\frac{\Delta T}{r}
$$

The uncertainty on $T_{c}$ and $T_{i n}$ being related to the measure of $r_{c}$ and $r_{i n}$, we get a $\pm 1.5{ }^{\circ} \mathrm{C}$ confidence interval on their values.

\section{APPENDIX B: VELOCITIES MEASURED FOR OTHER VOLTAGES}

We show here the droplets velocities $v_{r}$ measured by PIV (Fig. 10(a)) and the inner cloud radius propagating speed $\partial_{t} r_{i n}$ (Fig. 10(b)) for three other voltages: 7.9, 8.6 and 9.2 Volts.

At a constant room temperature, the higher the voltage applied, the higher the temperature gradient, and the higher the droplets velocities. Same as in Fig. 6, for each voltage the velocity profile is attenuated as the distance to the 

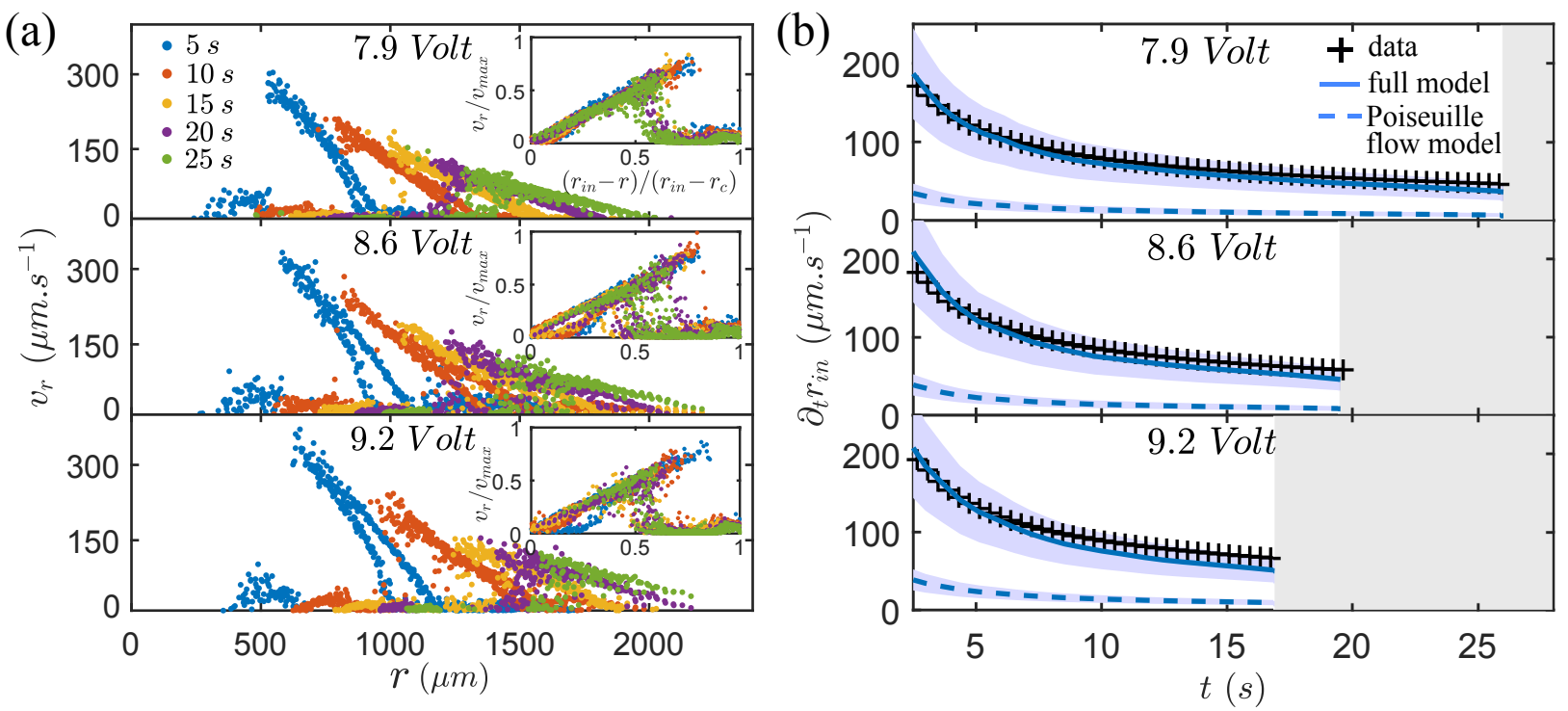

FIG. 10. (a) Velocity $v_{r}$ of the droplets coming back to the center versus the radial coordinate, at five successive times 5 , 10 , 15, 20 and $25 \mathrm{~s}$, for three voltages: $7.9,8.6$ and 9.2 Volt. The mass fraction in IL is $25 \%$, the cavity height is $H=60 \mu \mathrm{m}$. Inset: rescaled velocity versus the reduced spatial coordinate. All profiles collapse on a line with a slope of 1 . (b) Measured velocity of the cloud inner radius $\partial_{t} r_{i n}$ versus time, at 7.9, 8.6 and 9.2 Volt (black crosses). The blue dashed line represents the average velocity of the Poiseuille contribution eq.(2). The blue solid line is the sum of the Poiseuille and thermocapillary contributions eq.(3). The blue shaded areas give a confidence interval related to a $30 \%$ variation in $h$ and in $R$. The grey shaded zone on the right is out of the observation window $(r>2500 \mu \mathrm{m})$. The mass fraction in IL is $25 \%$, the cavity height is $H=60 \mu \mathrm{m}$.

center increases and the rescaled profiles collapse on a single line.

The propagation of $r_{i n}$ increases also with the voltage: both the counter-Poiseuille flow and the thermocapillary actuation see their contributions rise with the temperature gradient intensity. Our model follows well the data for the three voltages respectively.

\section{APPENDIX C: INTERFEROMETRIC DETECTION OF THE WETTING FILM}

The thickness variation of the wetting film is difficult to quantify with confocal experiments, but interferometric methods allow us to obtain at least one relative variation in height between two points [59]. We repeated the separation experiment with quasi-monochromatic illumination (mercury lamp filtered at $\lambda=546 \mathrm{~nm}$ ) and a magnification x10 (against $\mathrm{x} 5$ usually). By playing with the contrast of the recorded movies, the interference fringes present at the liquidliquid interface of the film become visible. Fig. 11 shows three successive stages of separation under monochromatic illumination.

Four dark fringes are visible and propagate radially following the same dynamics as the separation pattern. The inter-fringe distance decreases by $20 \%$ between the first and the third image. The difference in film thickness between two dark fringes is given by the formula:

$$
\Delta h_{\text {interfringe }}=\frac{\lambda}{2 n_{\text {film }}}
$$

with $n_{\text {film }}$ the optical index of the liquid forming the wetting film. We measured independently and at room temperature the optical index of our ionic liquid solutions using a pocket refractometer (ATAGO 3850 PAL-RI). The measurements are shown in figure 12. Assuming the composition of the IL-rich wetting film is about $60 w t \%$, we use $n_{\text {film }} \approx 1.40$ to calculate the thickness variation:

$$
\Delta h_{\text {interfringe }} \approx \frac{546}{2 \times 1.40} \approx 200 \mathrm{~nm}
$$

Thus the film sees its thickness vary by $\pm 800 \mathrm{~nm}$ along the radial coordinate. The wetting film is not completely flat but this micrometric variation of the thickness over $1 \mathrm{~mm}$ in length shows the very small curvature of the interface 


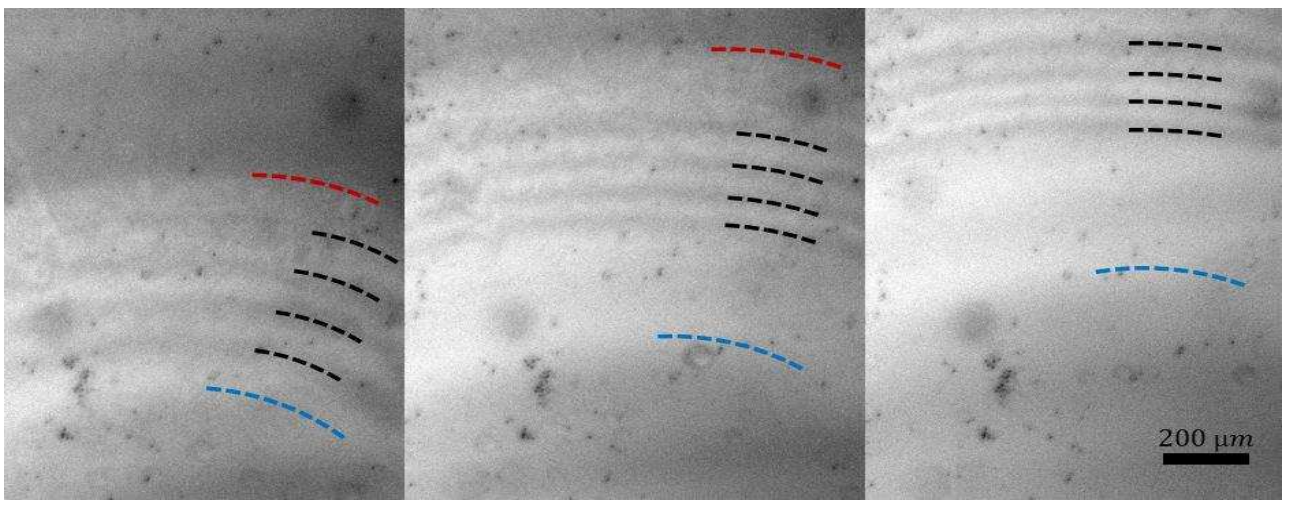

FIG. 11. Phase separation at three consecutive times (12, 18 and $26 \mathrm{~s})$ under monochromatic illumination $(\lambda=546 \mathrm{~nm})$. The four concentric fringes due to the film are pointed out by the black hatched arcs. The blue and red hatched arcs indicate $r_{c}$ and $r_{i n}$ respectively. The cavity height is $H=80 \mu \mathrm{m}$.

and allows us to assume a 2D Stokes equation in the lubrication approximation. This small time-dependent curvature of the film is reminiscent of previous works about the Marangoni-driven spreading of water-ethanol mixtures on a clean glass [60].

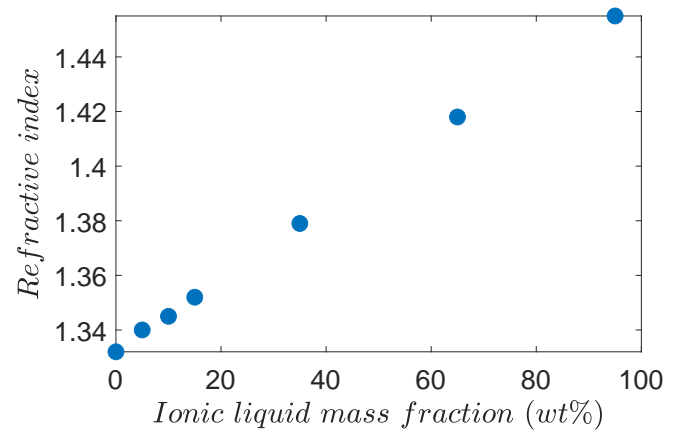

FIG. 12. Optical index of the water - ionic liquid mixture at $22^{\circ} \mathrm{C}$ as a function of its composition.

[1] David Dupont and Koen Binnemans, "Rare-earth recycling using a functionalized ionic liquid for the selective dissolution and revalorization of y 2 o 3: Eu 3+ from lamp phosphor waste," Green Chemistry 17, 856-868 (2015).

[2] Daphne Depuydt, Liwang Liu, Christ Glorieux, Wim Dehaen, and Koen Binnemans, "Homogeneous liquid-liquid extraction of metal ions with non-fluorinated bis (2-ethylhexyl) phosphate ionic liquids having a lower critical solution temperature in combination with water," Chemical Communications 51, 14183-14186 (2015).

[3] Yuki Kohno, Shohei Saita, Kenichi Murata, Nobuhumi Nakamura, and Hiroyuki Ohno, "Extraction of proteins with temperature sensitive and reversible phase change of ionic liquid/water mixture," Polymer Chemistry 2, 862-867 (2011).

[4] Douglas R MacFarlane, Alison L Chong, Maria Forsyth, Mega Kar, R Vijayaraghavan, Anthony Somers, and Jennifer M Pringle, "New dimensions in salt-solvent mixtures: a 4th evolution of ionic liquids," Faraday Discussions 206, 9-28 (2017).

[5] Yunxiang Qiao, Wenbao Ma, Nils Theyssen, Chen Chen, and Zhenshan Hou, "Temperature-responsive ionic liquids: fundamental behaviors and catalytic applications," Chemical Reviews 117, 6881-6928 (2017).

[6] Hiroyuki Ohno, Masahiro Yoshizawa-Fujita, and Yuki Kohno, "Functional design of ionic liquids: Unprecedented liquids that contribute to energy technology, bioscience, and materials sciences," Bulletin of the Chemical Society of Japan 92, 852-868 (2019).

[7] Chih-Hao Hsu, Canghai Ma, Ngoc Bui, Zhuonan Song, Aaron D Wilson, Robert Kostecki, Kyle M Diederichsen, Bryan D McCloskey, and Jeffrey J Urban, "Enhanced forward osmosis desalination with a hybrid ionic liquid/hydrogel thermoresponsive draw agent system," ACS Omega 4, 4296-4303 (2019).

[8] Eiji Kamio, Aki Takenaka, Tomoki Takahashi, and Hideto Matsuyama, "Fundamental investigation of osmolality, thermoresponsive phase diagram, and water-drawing ability of ionic-liquid-based draw solution for forward osmosis membrane process," Journal of Membrane Science 570, 93-102 (2019). 
[9] Yuki Deguchi, Nobuhumi Nakamura, and Hiroyuki Ohno, "Thermoresponsive ionic liquid/water mixtures for separation and purification technologies," Separation and Purification Technology 251, 117286 (2020).

[10] Yufeng Cai, Wenming Shen, Jing Wei, Tzyy Haur Chong, Rong Wang, William B Krantz, Anthony G Fane, and Xiao $\mathrm{Hu}$, "Energy-efficient desalination by forward osmosis using responsive ionic liquid draw solutes," Environmental Science: Water Research \& Technology 1, 341-347 (2015).

[11] Yujiang Zhong, Xinbo Wang, Xiaoshuang Feng, Selvedin Telalovic, Yves Gnanou, Kuo-Wei Huang, Xiao Hu, and Zhiping Lai, "Osmotic heat engine using thermally responsive ionic liquids," Environmental Science \& Technology 51, 9403-9409 (2017).

[12] Akira Onuki, "Dynamic van der waals theory," Physical Review E 75, 036304 (2007).

[13] JK Platten and G Chavepeyer, "Phase separation under a temperature gradient," Physica A: Statistical Mechanics and its Applications 213, 110-117 (1995).

[14] T Araki and H Tanaka, "Hydrodynamic delocalization of phase separation in a locally cooled fluid mixture," EPL (Europhysics Letters) 65, 214 (2004).

[15] Michael R Moldover and John W Cahn, "An interface phase transition: complete to partial wetting," Science 207, 10731075 (1980).

[16] D Beysens and D Esteve, "Adsorption phenomena at the surface of silica spheres in a binary liquid mixture," Physical Review Letters 54, 2123 (1985).

[17] Sanjay Puri, "Surface-directed spinodal decomposition," Journal of Physics: Condensed Matter 17, R101 (2005).

[18] Hajime Tanaka, "Interplay between phase separation and wetting for a polymer mixture confined in a two-dimensional capillary: wetting-induced domain ordering and coarsening," EPL (Europhysics Letters) 24, 665 (1993).

[19] Hajime Tanaka, "Dynamic interplay between phase separation and wetting in a binary mixture confined in a onedimensional capillary," Physical Review Letters 70, 53 (1993).

[20] Hajime Tanaka, "Wetting dynamics in a confined symmetric binary mixture undergoing phase separation," Physical Review Letters 70, 2770 (1993).

[21] Hajime Tanaka, "Double phase separation in a confined, symmetric binary mixture: Interface quench effect unique to bicontinuous phase separation," Physical Review Letters 72, 3690 (1994).

[22] Hajime Tanaka and Tomoo Sigehuzi, "Spinodal decomposition of a symmetric binary fluid mixture in quasi two dimensions: Local orientational ordering of fluid tubes," Physical Review E 52, 829 (1995).

[23] Kurt Binder, "Spinodal decomposition in confined geometry," Journal of Non Equilibrium Thermodynamics 23, 1-44 (1998).

[24] Hajime Tanaka, "Interplay between wetting and phase separation in binary fluid mixtures: roles of hydrodynamics," Journal of Physics: Condensed Matter 13, 4637 (2001).

[25] Ludovic Keiser, Rémy Herbaut, José Bico, and Etienne Reyssat, "Washing wedges: capillary instability in a gradient of confinement," Journal of Fluid Mechanics 790, 619-633 (2016).

[26] AM Cazabat, F Heslot, SM Troian, and P Carles, "Fingering instability of thin spreading films driven by temperature gradients," Nature 346, 824-826 (1990).

[27] Vincent Miralles, Bertrand Selva, Isabelle Cantat, and M-C Jullien, "Foam drainage control using thermocapillary stress in a two-dimensional microchamber," Physical Review Letters 112, 238302 (2014).

[28] Mark Menesses, Matthieu Roché, Laurent Royon, and James C Bird, "Surfactant-free persistence of surface bubbles in a volatile liquid," Physical Review Fluids 4, 100506 (2019).

[29] G Koleski, A Vilquin, J-C Loudet, T Bickel, and B Pouligny, "Azimuthal instability of the radial thermocapillary flow around a hot bead trapped at the water-air interface," Physics of Fluids 32, 092108 (2020).

[30] NO Young, JS Goldstein, and Mi J Block, "The motion of bubbles in a vertical temperature gradient," Journal of Fluid Mechanics 6, 350-356 (1959).

[31] Alois Würger, "Thermally driven marangoni surfers," arXiv preprint arXiv:1407.6818 (2014).

[32] Takeaki Araki and Anna Maciołek, "Illumination-induced motion of a janus nanoparticle in binary solvents," Soft Matter 15, 5243-5254 (2019).

[33] Juan Ruben Gomez-Solano, Sutapa Roy, Takeaki Araki, S. Dietrich, and Anna Macioek, "Transient coarsening and the motility of optically heated janus colloids in a binary liquid mixture," Soft Matter 16, 8359-8371 (2020).

[34] Thomas Zinn, Lewis Sharpnack, and Theyencheri Narayanan, "Phoretic dynamics of colloids in a phase separating critical liquid mixture," Physical Review Research 2, 033177 (2020).

[35] Kilian Dietrich, Nick Jaensson, Ivo Buttinoni, Giorgio Volpe, and Lucio Isa, "Microscale marangoni surfers," Physical Review Letters 125, 098001 (2020).

[36] Daniel Beysens, Yves Garrabos, VS Nikolayev, Carole Lecoutre-Chabot, J-P Delville, and John Hegseth, "Liquid-vapor phase separation in a thermocapillary force field," EPL (Europhysics Letters) 59, 245 (2002).

[37] Akira Onuki and Kentaro Kanatani, "Droplet motion with phase change in a temperature gradient," Physical Review E 72, 066304 (2005).

[38] Vadim S Nikolayev, Yves Garrabos, Carole Lecoutre, Guillaume Pichavant, Denis Chatain, and Daniel Beysens, "Evaporation condensation-induced bubble motion after temperature gradient set-up," Comptes Rendus Mécanique 345, 35-46 (2017).

[39] Michel Assenheimer, Boris Khaykovich, and Victor Steinberg, "Phase separation of a critical binary mixture subjected to a temperature gradient," Physica A: Statistical Mechanics and its Applications 208, 373-393 (1994).

[40] F Morick and D Woermann, "Convection generated by a gradient of interfacial tension in a phase separated binary liquid mixture with a miscibility gap," Zeitschrift für Physikalische Chemie 205, 1-14 (1998). 
[41] Margaux Kerdraon, Joshua D McGraw, Benjamin Dollet, and Marie-Caroline Jullien, "Self-similar relaxation of confined microfluidic droplets," Physical Review Letters 123, 024501 (2019).

[42] Yuki Kohno, Hiroki Arai, Shohei Saita, and Hiroyuki Ohno, "Material design of ionic liquids to show temperature-sensitive lcst-type phase transition after mixing with water," Australian Journal of Chemistry 64, 1560-1567 (2012).

[43] Ayako Nitta, Takeshi Morita, Keiko Nishikawa, and Yoshikata Koga, "Mixing scheme of an aqueous solution of tetrabutylphosphonium trifluoroacetate in the water-rich region," Physical Chemistry Chemical Physics 19, 16888-16896 (2017).

[44] Carlos A Grattoni, Richard A Dawe, C Yen Seah, and Jane D Gray, "Lower critical solution coexistence curve and physical properties (density, viscosity, surface tension, and interfacial tension) of 2, 6-lutidine+ water," Journal of Chemical and Engineering Data 38, 516-519 (1993).

[45] Bertrand Selva, Julien Marchalot, and Marie-Caroline Jullien, "An optimized resistor pattern for temperature gradient control in microfluidics," Journal of Micromechanics and Microengineering 19, 065002 (2009).

[46] Eric D Siggia, "Late stages of spinodal decomposition in binary mixtures," Physical Review A 20, 595 (1979).

[47] Daniel A Beysens, "Kinetics and morphology of phase separation in fluids: The role of droplet coalescence," Physica A: Statistical Mechanics and its Applications 239, 329-339 (1997).

[48] DGAL Aarts and HNW Lekkerkerker, "Confocal scanning laser microscopy on fluid-fluid demixing colloid-polymer mixtures," Journal of Physics: Condensed Matter 16, S4231 (2004).

[49] William Thielicke and Eize Stamhuis, "Pivlab-towards user-friendly, affordable and accurate digital particle image velocimetry in matlab," Journal of Open Research Software 2 (2014).

[50] David Rivière, Bertrand Selva, Hamza Chraibi, Ulysse Delabre, and Jean-Pierre Delville, "Convection flows driven by laser heating of a liquid layer," Physical Review E 93, 023112 (2016).

[51] B Selva, L Daubersies, and J-B Salmon, "Solutal convection in confined geometries: Enhancement of colloidal transport," Physical Review Letters 108, 198303 (2012).

[52] Matthieu Roché, Zhenzhen Li, Ian M Griffiths, Sébastien Le Roux, Isabelle Cantat, Arnaud Saint-Jalmes, and Howard A Stone, "Marangoni flow of soluble amphiphiles," Physical Review Letters 112, 208302 (2014).

[53] Jinnan Chen and Kathleen J Stebe, "Surfactant-induced retardation of the thermocapillary migration of a droplet," Journal of Fluid Mechanics 340, 35-59 (1997).

[54] D Beysens and T Narayanan, "Wetting-induced aggregation of colloids," Journal of Statistical Physics 95, 997-1008 (1999).

[55] Marc Pascual, Margaux Kerdraon, Quentin Rezard, Marie-Caroline Jullien, and Lorène Champougny, "Wettability patterning in microfluidic devices using thermally-enhanced hydrophobic recovery of pdms," Soft Matter 15, 9253-9260 (2019).

[56] Dhiraj Nandyala, Zhen Wang, David Hwang, Thomas Cubaud, and Carlos E Colosqui, "Design, fabrication, and analysis of a capillary diode for potential application in water-oil separation," ACS Applied Materials \& Interfaces 12, 45950-45960 (2020).

[57] Prasenjit Das, Prabhat K Jaiswal, and Sanjay Puri, "Surface-directed spinodal decomposition on chemically patterned substrates," Physical Review E 102, 012803 (2020).

[58] Prasenjit Das, Prabhat K Jaiswal, and Sanjay Puri, "Surface-directed spinodal decomposition on morphologically patterned substrates," Physical Review E 102, 032801 (2020).

[59] Ludovic Keiser, Hadrien Bense, Pierre Colinet, J Bico, and Etienne Reyssat, "Marangoni bursting: evaporation-induced emulsification of binary mixtures on a liquid layer," Physical Review Letters 118, 074504 (2017).

[60] JB Fournier and AM Cazabat, "Tears of wine," EPL (Europhysics Letters) 20, 517 (1992). 